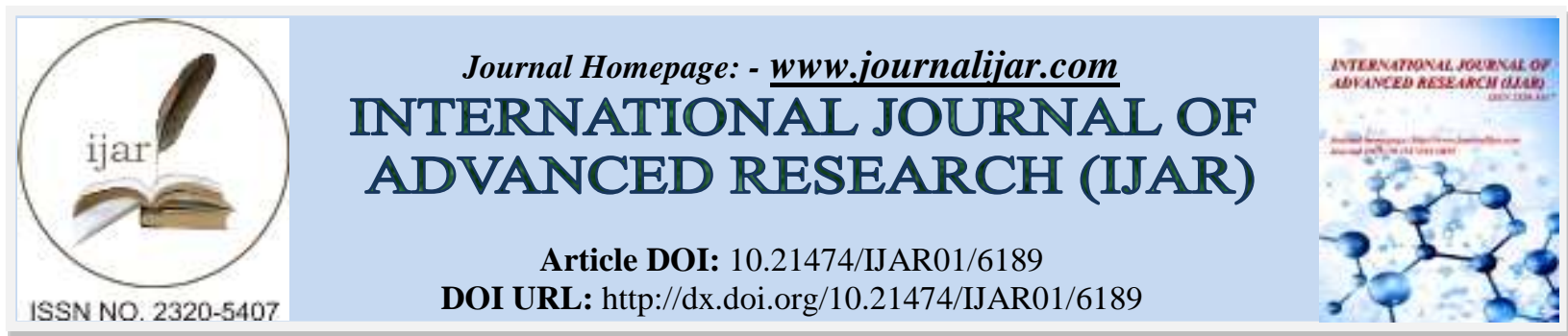

RESEARCH ARTICLE

\title{
EARLY CLINICAL RESULTS OF MODULAR BIPOLAR HEMIARTHROPLASTY FOR FRACTURES OF FEMORAL NECK.
}

\section{Dr. Jabreel Muzaffar ${ }^{1}$, Dr. Jawahar Mehmood khan ${ }^{2}$, Dr. Sumit Kumar ${ }^{3}$, Dr Mohd Bilal Kaleem ${ }^{4}$ and Dr} Ariz Nawaz Khan ${ }^{5}$.

1. SR Department of orthopaedics Shri Mahant Indresh Hospital Dehradun.

2. JR Department of orthopaedics GMC Jammu.

3. JR Department of orthopaedics Shri Mahant Indresh Hospital Dehradun.

4. JR Shri Mahant Indresh Hospital Dehradun.

\section{Manuscript Info}

Manuscript History

Received: 02 November 2017

Final Accepted: 04 December 2017

Published: January 2018

\begin{abstract}
Fractures of neck of femur are commonly seen in elderly people although all age groups can be affected, but increased predilection is seen in osteoporotic bones. The objective of treatment of femoral neck fractures in the mobile elderly population is early restoration of premorbid walking ability and quality of life. . Hemi or total hip arthroplasty is an accepted treatment of fracture neck of femur in the elderly. This study was carried out in the Department of Orthopaedics, Govt. Medical College Jammu during the period of August 2015 to December 2016. Patients operated till June 2016 were included in the study. All the patients who presented in the Department with intracapsular fractures of neck of femur were classified according to Garden's Classification and those who meet the eligibility criteria were included in the study. The mean age was 72.4 years. The range of age was $61-88$ years. There were $12(40 \%)$ male cases and $18(60 \%)$ female cases (sex ratio 1:1.5). There were 18 (60\%) Garden's type IV fractures and $12(40 \%)$ Garden's type III fractures. All patients were followed for period of 12 months. The average Harris Hip Score was 81.26(range 69-91). $76.67 \%$ of patients achieved good to excellent results. $16.66 \%$ patients had fair outcome. 2 patients had poor results. The treatment of these commonly found fractures with modular bipolar hemiarthroplasty has given promising results. The short term outcome from this study provides evidence that this is suitable treatment option in displaced intracapsular fracture neck of femur in elderly.
\end{abstract}

Copy Right, IJAR, 2018,. All rights reserved.

\section{Introduction:-}

Fractures of neck of femur are commonly seen in elderly people although all age groups can be affected, but increased predilection is seen in osteoporotic bones (Aitken, 1984). The best treatment for the active and lucid elderly patient with displaced intra-capsular femoral neck fracture is still controversial and has been rightly called "Unsolved Fracture" by Dickson (1953). Due to high energy road traffic accidents the fracture have presented in young patients further complicating the establishment of definitive treatment. 
The objective of treatment of femoral neck fractures in the mobile elderly population is early restoration of premorbid walking ability and quality of life. Hemi or total hip arthroplasty is an accepted treatment of fracture neck of femur in the elderly.

With the introduction of modular hemiarthroplasty whether unipolar or bipolar the neck length can be adjusted with regards to tension of abductors and leg length and further it can more easily be converted to Total Hip Replacement.

\section{Materials and Methods:-}

This study was carried out in the Department of Orthopaedics, Govt. Medical College Jammu during the period of August 2015 to December 2016. Patients operated till June 2016 were included in the study. All the patients who presented in the Department with intracapsular fractures of neck of femur were classified according to Garden's Classification and those who met the eligibility criteria were included in study.

\section{Inclusion Criteria:-}

1. Age $>60$ years.

2. Garden's type III and type IV fractures.

3. Pre-injury independent walking capability with or without aids.

4. Both fresh and old fractures were included.

5. Nonunion of fracture neck femur.

6. Avascular necrosis of femoral head following prior treatment.

7. Implant failure.

8. Abbreviated Mental Test Score $\geq 6$ (Hodkinson HM, 1972).

\section{Exclusion Criteria:-}

1. Un-displaced Fractures (Garden's type I and II).

2. Patients with pre-existing joint diseases like Rheumatoid athritis, Osteoarthritis.

3. Patients with Pathological Fractures, Multiple fractures.

4. Pre-injury non ambulatory patients.

5. Abbreviated Mental Test Score (AMTS) $<6$.

6. Patients with medical contraindications for surgery/ anaesthesia.

7. Psychiatry patients with mental instability.

Patients were assessed for fitness for surgery by an anaesthetist. The patients consent for participation in the study was obtained. Once the patient was considered fit for the surgery, he/she was taken for surgery. Detailed clinical and radiological examinations with other relevant investigations were carried out in all the patients in orthopaedic emergency/indoor/OPD.

Prophylactic antibiotic: All patients were given 1.5gcefuroxime 1/2hour before surgery.

Surgical approach: The operation was performed by the exposing the hip through Direct lateral approach described by (Hardinge, 1982).

Postoperatively patients were carefully monitored with respect to vitals and position of limb. Limb was placed in abduction $\left(15^{\circ}\right)$ and neutral rotation. Antibiotics were administered in appropriate doses along with analgesics as per need of the patient. Parental antibiotics were continued till 5th postoperative day and switched over to oral antibiotics if the drain culture was negative and wound showed no signs of infection for five days. Active isometric quadriceps, isometric gluteal, ankle pumps and deep breathing exercises were started on $1^{\text {st }}$ Post-op day. On the second postoperative day, the patient was made to sit on the side of the bed or in a semi-recumbent position. Drain was removed after 24 hours and its tip sent for culture and sensitivity. The patient's second dressing was done on $5^{\text {th }}$ post op day. Patients were allowed to partially bear weight with the help of walker as pain permitted after $2^{\text {nd }}$ post op day if the wound was uncomplicated. By $12^{\text {th }}$ Post-op day stitches were removed. The patients were discharged after second dressing if wound was healthy with the advice to follow a prescribed home care program which included the following:

1. No squatting or sitting cross legged.

2. Use of Western type toilet (commode).

3. Regular quadriceps, hamstring and abduction exercises of hips were advised. 
4. Walk with the aid of walker.

5. To have an attendant accompanying while walking.

6. Don't sit with knees higher than hips and shoulders forward than pelvis.

7. Keep pillow in between thighs while sleeping CASE 1

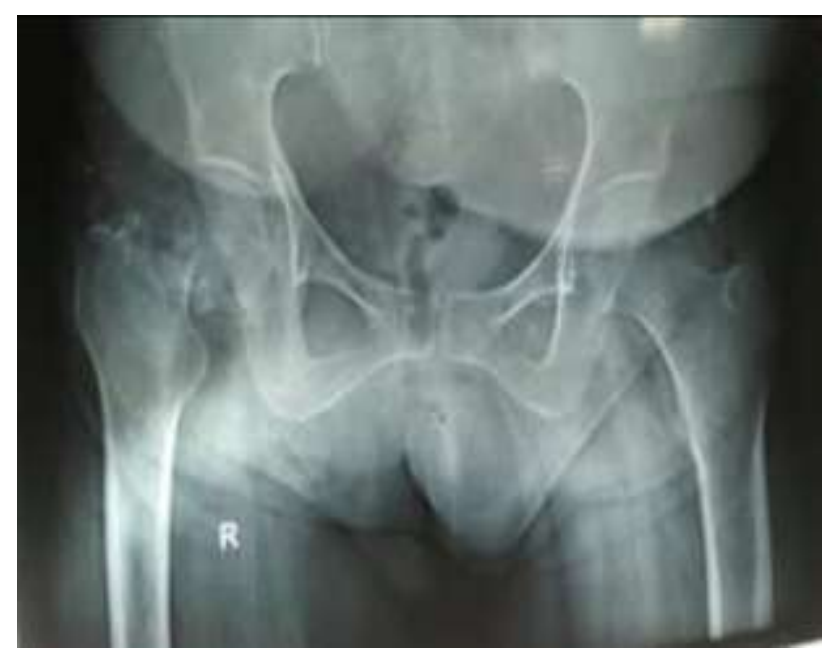

Preoperative X-Ray

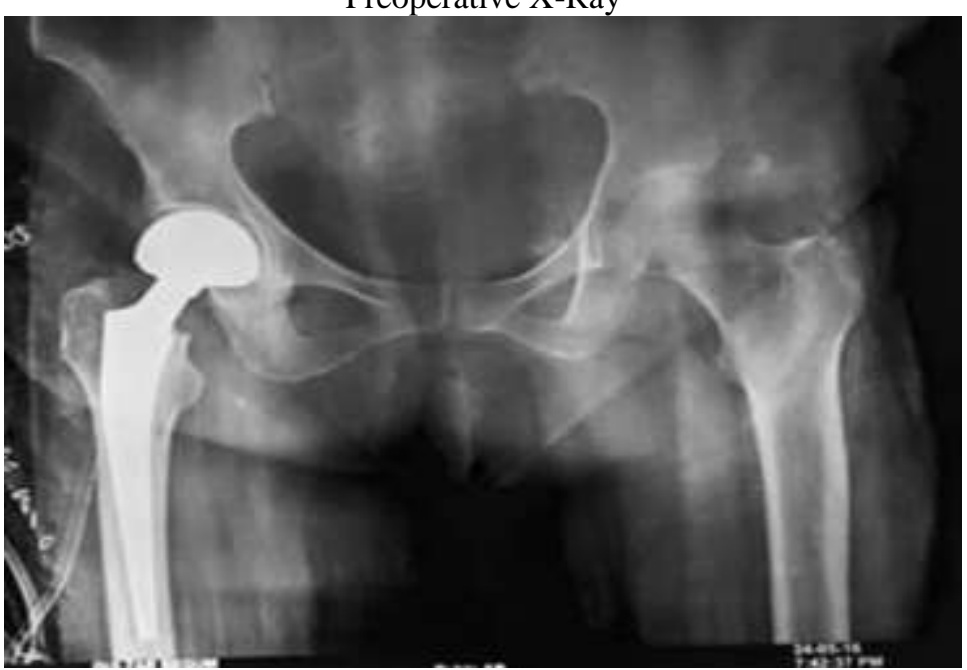

Immediate Postoperative X-Ray

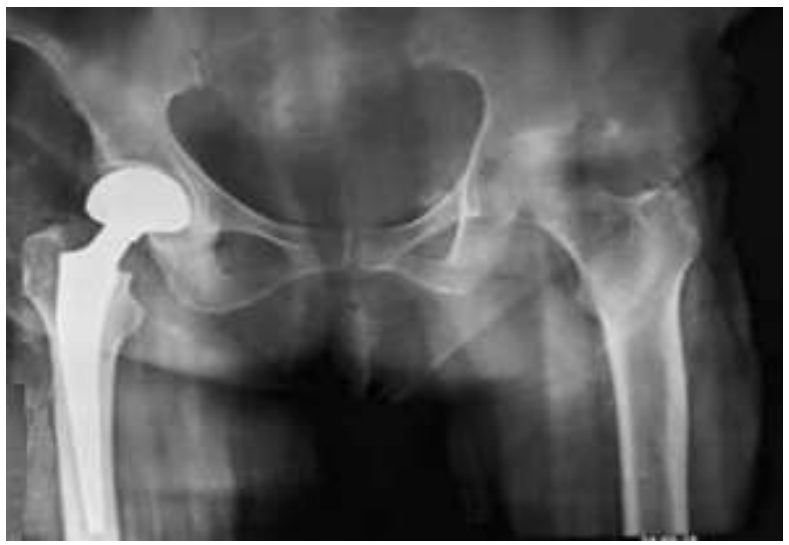

12 Months Postoperative X-Ray 


\section{CASE 2}

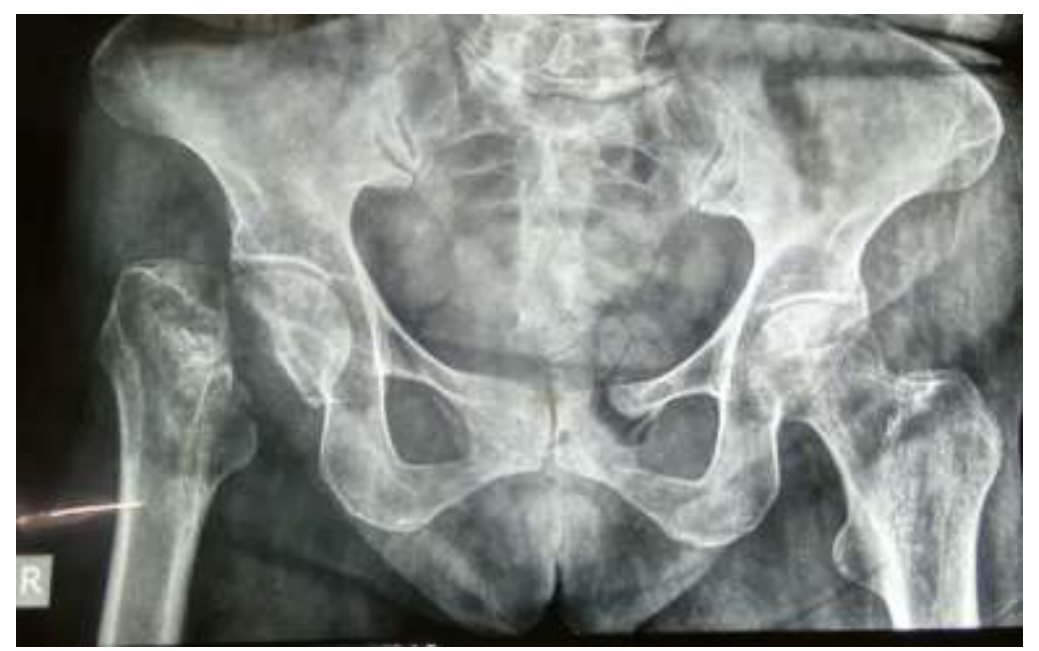

Preoperative X-Ray

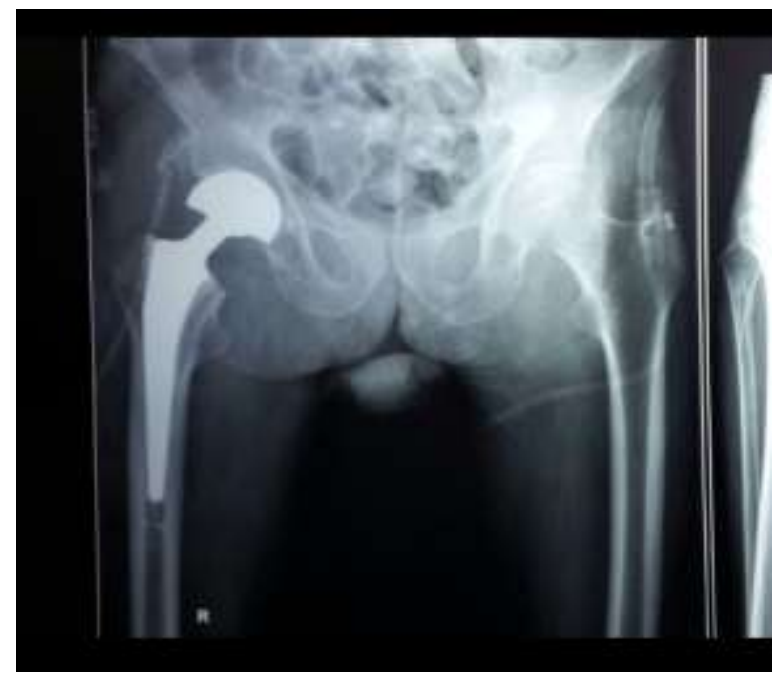

Immediate Postoperative X-Ray

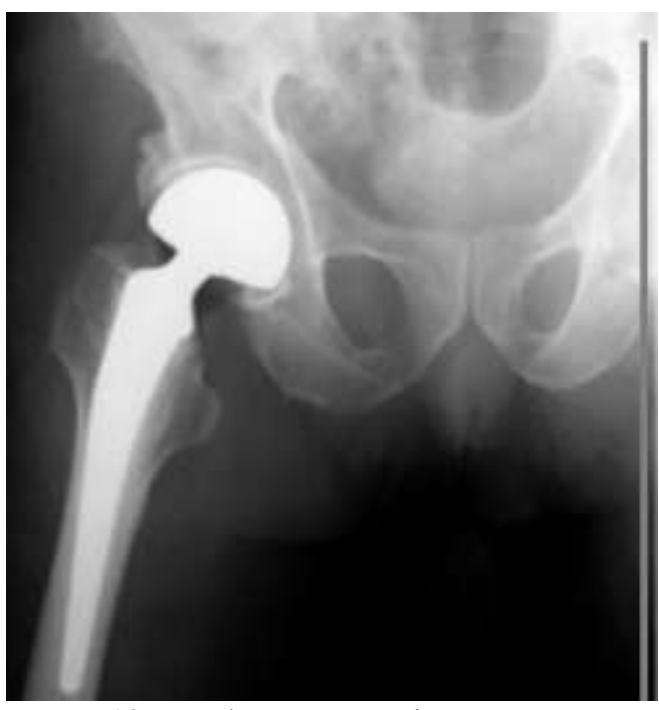

12 Months Postoperative X-Ray 
Partial weight bearing as tolerated with walker for at least 3 weeks was advised, followed by partial weight bearing with cane followed by full weight bearing by six weeks.

The patients were advised to attend the orthopaedic OPD regularly. The patients were evaluated during each follow up at 6 weeks, three months, and 6 months post operatively using Modified Harris Hip score (William $\mathrm{H}$ Harris,1969). Fracture neck of femur and its complications continue to be the unsolved problem and still pose a major challenge to an orthopaedic surgeon. In spite of many technical advancements controversy still exists in ideal treatment of fracture neck of femur in elderly. In view of this conflicting background this short term study of hemiarthroplasty with modular bipolar prosthesis was taken up in 30 elderly patients.

\section{Results:-}

Age incidence: 9 cases (30\%) were in the age group of $60-70$ years and $18(60 \%)$ in age group of $70-80$ years. There were 3 cases in age group of 80 - 90 years. The mean age was 72.4 years. The range of age was $61-88$ years. Sex Distribution: There were 12 (40\%) male cases and 18 (60\%) female cases (sex ratio 1:1.5).

Type of Fracture: There were 18 (60\%) Garden's type IV fractures and 12 (40\%) Garden's type III fractures. Ratio of Garden's type Ill to type IV was 1:1.35.

Mode of Injury: The predominant mode of injury was fall on ground (slip), 90\%. RTA was the cause of fracture in 3 patients $10 \%$.

Associated illnesses: Out of all these patients 9 patients were Hypertensive. 3 patients were having diabetes mellitus. Bronchial asthma was present in 2 patients.

Size of Femoral Head: The size of femoral head ranged from 43 to $53 \mathrm{~mm}$. The most common size was $47 \mathrm{~mm}$ (20\%). The mean femoral head diameter was $47.23 \mathrm{~mm}$ (range $43-53 \mathrm{~mm}$ ).

Blood Loss: The average blood loss was $256.33 \mathrm{ml}$ (range 150 - $450 \mathrm{ml})$.

Duration of Surgery: The average duration of surgery was $66.55 \mathrm{~min}($ range $50-90 \mathrm{~min}$ ).

Follow up: All patients were followed for period of 12 months.

Harris Hip Score. The average Harris Hip Score was 81.26(range 69-91). 76.67\% of patients achieved good to excellent results. $16.66 \%$ patients had fair outcome. 2 patients had poor results.

Table:- Grading Of Result (Modified Harris Hip Score)

\begin{tabular}{|l|l|l|l|}
\hline Result & Points & No of cases & Percentage \\
\hline Excellent & $90-100$ & 2 & 6.67 \\
\hline Good & $80-89$ & 21 & 76.66 \\
\hline Fair & $70-79$ & 5 & 16.67 \\
\hline Poor & $<70$ & 2 & 0 \\
\hline
\end{tabular}

Complications: Superficial Wound Infection occurred in 2 cases (6.67\%). It cleared with dressings and appropriate antibiotic therapy after culture and sensitivity. The patients were managed by local dressings and using water mattress. There was no cases of limb length discrepancy, fixed deformities foot drop, sciatic nerve palsy, mortality, deep infection DVT, heterotopic ossification in our series.

\section{Discussion:-}

The treatment of femoral neck fractures in the mobile elderly is directed at rapid restoration of preinjury functional and ambulatory status. Algorithms for femoral neck fracture treatment have been defined. Leighton $e t$ $a l$. recommend prosthetic replacement for patients more than 60 years old and having a displaced femoral neck fracture.

Postoperative mortality following hip replacements is usually due to cardiopulmonary causes (myocardial infarction or pulmonary emboli). ${ }^{1}$ Intraoperative deaths (cardiac arrest) during hip arthroplasty occur infrequently and have 
been associated with bone cement (BCIS). ${ }^{7,8}$ Patients with severe underlying cardiovascular In our series, the average operative time was $66.55 \mathrm{~min}$ (range 50-90min) and average blood loss $256.33 \mathrm{ml}$ (range: 150-450 ml). There was no intraoperative or immediate postoperative mortality. This may be due in part to the excellent postoperative care and intensive care setup we had available. . The average Harris Hip Score was 81.26(range 6991). $76.67 \%$ of patients achieved good to excellent results. $16.66 \%$ patients had fair outcome. 2 patients had poor results. There was no cases of dislocation, fixed deformities foot drop, sciatic nerve palsy, mortality, deep infection DVT, heterotopic ossification in our series. There was no case of limb length discrepancy, because of greater scope of adjusting the length on operation table due to availability of all sizes of stem, neck and head which were adjusted to suitable length, that being greatest advantage of using modular system. We have assessed our results without standardizing the different variables responsible (viz. age, gender, fracture classification, osteoporosis, etc.), and this may be one of the drawbacks of our study. Most of the studies between modern modular or bipolar components vs Austin Moore or a Thompson's prosthesis show that the former are better because the neck length can be adjusted with regards to tension of abductors and leg length and further it can more easily be converted to Total Hip Replacement.

Hemiarthroplasty using Modular Bipolar prosthesis for fractures of the displaced femoral neck in elderly provides freedom from pain and rapid return to unassisted activity with an acceptable complication rate. Though conventionally done in cemented fashion, providing a good primary anchorage, especially in the osteoporotic femur is of paramount importance. The end functional results also depend on the associated co-morbidity and optimum postoperative rehabilitation. Throughout the purview of the present study, our experience with cemented Modular bipolar prosthesis has been better. . The short term outcome from this study provides evidence that this is suitable treatment option in displaced intracapsular fracture neck of femur in elderly. The long term results using Modular bipolar prosthesis needs further study for a longer period in a larger sample with a direct comparison between the cemented versus uncemented groups.

\section{Conclusion:-}

The treatment of these commonly found fractures with modular bipolar hemiarthroplasty has given promising results. The short term outcome from this study provides evidence that this is suitable treatment option in displaced intracapsular fracture neck of femur in elderly.

\section{References:-}

1. Aitken JM. Relevance of osteoporosis in women with fracture of femoral neck. BMJ 1984;288:597-60.

2. Byrick RJ. Cement implantation syndrome: A time limited embolic phenomenon. Can J Anaesth. 1997;44:10711.

3. Dickson JA. The unsolved fracture. J Bone Joint Surg 1984;35-A:805-822.

4. Donaldson AJ, Thomson HE, Harper NJ, Kenny NW. Bone cement implantation syndrome. Br J Anaesth. 2009;102:12-22.

5. Hardinge K. The direct lateral approach to the hip. J Bone Joint Surgery Br1982;64(1):17-9.

6. Harris WH. Traumatic arthritis of the hip after dislocation and acetabular fractures: treatment by mould arthroplasty. An end result study using a new method of result evaluation. J Bone Joint Surg Am 1969; 51(4):737-55. 6.

7. Hodkinson HM. Evaluation of mental test score for assessment of mental impairment in elderly. Age and Ageing1972; 1:233-238.

8. Leighton RK, Schmidt AH, Collier P, Trask K. Advances in the treatment of intracapsular hip fractures in the elderly. Injury. 2007;38:S24-34.

9. Parker MJ, Gurusamy K. Arthroplasties (with and without bone cement) for proximal femoral fractures in adults. Cochrane Database Syst Rev. 2006;3:CD001706.

10. Parvizi J, Holiday AD, Ereth MH, Lewallen DG. Sudden death during primary hip arthroplasty. Clin Orthop Relat Res. 1999;369:39-48. 